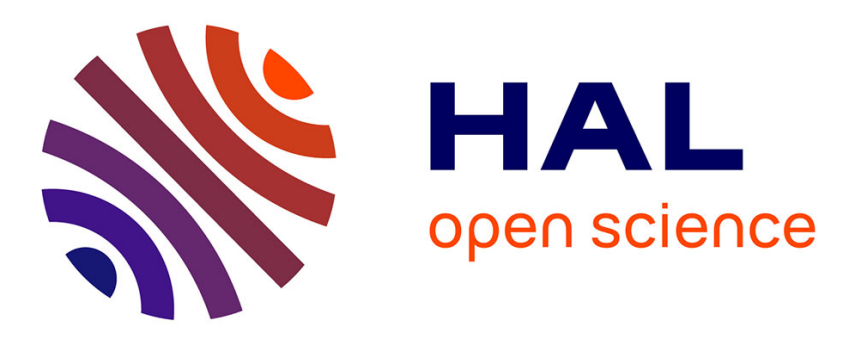

\title{
A scatter search heuristic for maximizing the net present value of a resource-constrained project with fixed activity cash flows
}

\author{
Mario Vanhoucke
}

\section{- To cite this version:}

Mario Vanhoucke. A scatter search heuristic for maximizing the net present value of a resourceconstrained project with fixed activity cash flows. International Journal of Production Research, 2010, 48 (07), pp.1983-2001. 10.1080/00207540802010781 . hal-00565116

\author{
HAL Id: hal-00565116 \\ https://hal.science/hal-00565116
}

Submitted on 11 Feb 2011

HAL is a multi-disciplinary open access archive for the deposit and dissemination of scientific research documents, whether they are published or not. The documents may come from teaching and research institutions in France or abroad, or from public or private research centers.
L'archive ouverte pluridisciplinaire HAL, est destinée au dépôt et à la diffusion de documents scientifiques de niveau recherche, publiés ou non, émanant des établissements d'enseignement et de recherche français ou étrangers, des laboratoires publics ou privés. 


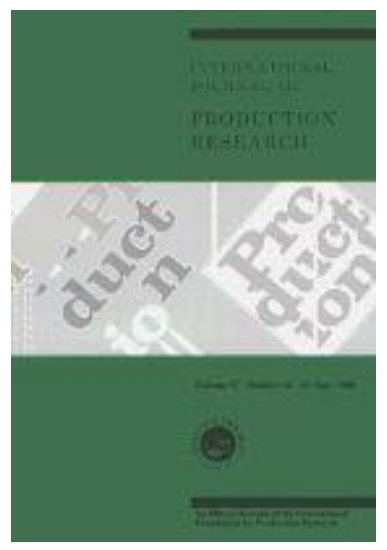

A scatter search heuristic for maximizing the net present value of a resource-constrained project with fixed activity cash flows

\begin{tabular}{|r|l|}
\hline Journal: & International Journal of Production Research \\
\hline Manuscript ID: & TPRS-2007-IJPR-0239.R2 \\
\hline Manuscript Type: & Original Manuscript \\
\hline $\begin{array}{r}\text { Date Submitted by the } \\
\text { Author: }\end{array}$ & 13-Dec-2007 \\
\hline Complete List of Authors: & $\begin{array}{l}\text { Vanhoucke, Mario; Ghent University, Dept of Mgmt Info, Ops Mgmt } \\
\text { and Tech Policy }\end{array}$ \\
\hline Keywords: & EVOLUTIONARY ALGORITHMS, PROJECT SCHEDULING \\
\hline Keywords (user): & EVOLUTIONARY ALGORITHMS, PROJECT SCHEDULING \\
\hline
\end{tabular}

\section{5cholarONE" \\ Manuscript Central}




\title{
A scatter search heuristic for maximizing the net present value of a resource-constrained project with fixed activity cash flows
}

\author{
Mario Vanhoucke $e^{1,2}$ \\ ${ }^{1}$ Faculty of Economics and Business Administration, Ghent University, Gent, Belgium \\ ${ }^{2}$ Operations \& Technology Management Centre, Vlerick Leuven Gent Management School, Gent, Belgium \\ mario.vanhoucke@ugent.beormario.vanhoucke@vlerick.be
}

In this paper, we present a meta-heuristic algorithm for the resource-constrained project scheduling problem with discounted cash flows. We assume fixed payments associated with the execution of project activities and develop a heuristic optimization procedure to maximize the net present value of a project subject to the precedence and renewable resource constraints.

We investigate the use of a bi-directional generation scheme and a recursive forward/backward improvement method from literature and embed them in a meta-heuristic scatter search framework. We generate a large dataset of project instances under a controlled design and report detailed computational results. The solutions and project instances can be downloaded from a website in order to facilitate comparison with future research attempts.

Keywords: Resource-constrained project scheduling; Net present value; Scatter search

$2^{\text {nd }}$ revised version December 2007 


\section{Introduction and problem formulation}

Project scheduling has been a research topic for many decades, resulting in a wide variety of optimization procedures. The main focus on the project duration minimization has led to the development of various exact and (meta-)heuristic procedures for resource-constrained project scheduling problems (RCPSP) under a wide variety of assumptions. For an overview of resource-constrained project scheduling in general, we refer to excellent overview papers of Brücker et al. (1999), Herroelen et al. (1998), Icmeli et al. (1993), Kolisch and Padman (2001) and Özdamar and Ulusoy (1995). Less, but not little, attention has been spent on the presence of financial aspects in project scheduling, leading to various optimization models where the net present value of the project, rather than the project duration, is the major objective. This problem formulation appears when a series of cash flows occur over time during project execution. The increasing attention on net present value maximization has led to the development of financial model formulations under various assumptions (positive and negative cash flows/time-dependent and -independent cash flows/single-mode versus multi-mode formulations/etc.). Despite the growing financial attention in project scheduling, little effort has been made to facilitate comparison between procedures as is the case in other domains. The latter can, for example, be illustrated in the competitive nature of research on the basic resource-constrained project scheduling problem (see e.g. Hartmann and Kolisch (2000) and Kolisch and Hartmann (2006)).

In this paper, the single-mode resource-constrained project șcheduling problem with discounted cash flows (RCPSPDC) is studied. This problem formulation is an extension of the basic RCPSP to the presence of activity cash flows, and assumes renewable resources with a constant availability and no activity preemption. We assume that all activity cash flows occur at predefined time points during execution of the corresponding activity, and hence, exclude more general problem formulations with, for example, progress payments, time-dependent cash flows or payments associated with events. We present a scatter search algorithm and test the new procedure on a large set of data instances. Computational results are reported and detailed information is uploaded on a website accessible by other researchers.

A project is represented by an activity-on-the-node network $\mathrm{G}=(\mathrm{N}, \mathrm{A})$, where the nodes in the set $\mathrm{N}$ represent the project activities and the arcs of set A the finish-start precedence relations with a time-lag of zero. The activities are numbered from a dummy start node 0 to a dummy end node $n+1$. Each activity $i$ has a duration $d_{i}$ and its performance involves a series of cash flow payments and receipts throughout this duration. When $c f_{i t}$ denotes the pre-specified cash flow of activity $i$ in period $t$ of its execution, a terminal value $c_{i}$ upon completion can be calculated by compounding $c f_{i t}$ to the end of the activity as $c_{i}=\sum_{t=1}^{d_{i}} c f_{i t} e^{\alpha\left(d_{i}-t\right)}$ with $\alpha$ the discount rate. If the non-negative integer variable $s_{i}$ represents the starting time activity $i$, its discounted value at the beginning of the project is $c_{i} e^{-\alpha\left(s_{i}+d_{i}\right)}$. Each activity requires $r_{i k}$ 
units of renewable resource $k$ which has a constant availability of $a_{k}$ units. Each project must be finished before a pre-specified project deadline $\delta_{n+1}$. The problem can be represented as $m, 1$ lcpm, $\delta_{n}, c_{j} \mid n p v$ following the classification scheme of Herroelen et al. (1999) or as PSlprecl $\sum C_{j}^{F} \beta^{C_{j}}$ following the classification scheme of Brücker et al. (1999) and is known to be NP-hard (Blazewicz et al. (1983)). A conceptual formulation for the RCPSPDC can be given as follows:

$$
\text { Maximize } \sum_{i=1}^{n} c_{i} e^{-\alpha\left(s_{i}+d_{i}\right)}
$$

Subject to

$$
\begin{array}{ll}
s_{i}+d_{i} \leq s_{j} & \forall(i, j) \in A \\
\sum_{i \in S(t)} r_{i k} \leq a_{k} & k=1, \ldots, K \text { and } t=1, \ldots, \delta_{n+1} \\
s_{n+1} \leq \delta_{n+1} &
\end{array}
$$

where $\mathrm{S}(\mathrm{t})$ denotes the set of activities in progress in period $] t-1, t]$.

Eq. [1] maximizes the net present value of the project. Eq. [2] takes the finish-start precedence relations with a time-lag of zero into account. The renewable resource constraints are satisfied thanks to eq. [3]. Eq. [4] imposes a hard pre-specified deadline to the project.

The resource-constrained project scheduling problem with discounted cash flows belongs to the class of NP-hard problems, and hence, many heuristic solution procedures have been developed and described in the literature. Many research papers focus on the development of single-pass algorithms in which activities are ranked by a priority vector determining the order of resource allocation during a schedule generation process. Since these methods can only generate a single solution, they are often extended by improvement methods and/or backward scheduling schemes. During the recent decade, increasing computer power has led to the development of various multi-pass algorithms, leading to resource-constrained project scheduling problem formulations under a wide variety of cash flows assumptions. In the current paper under study, we present a multi-pass scatter search heuristic (section 3) for the RCPSPDC under the fixed activity cash flow assumptions described earlier, which relies on a bi-directional generation scheme (sub-section 2.1) and a recursive forward/backward improvement step (sub-section 2.2). Mika et al. (2005) give an extensive literature overview of net present value maximization in project scheduling, and hence, it does not need to be repeated here.

The foundation for the current research paper has been laid by Selle and Zimmermann (2003) who have developed a so-called bi-directional schedule generation scheme for large-scaled RCPSPDC instances with generalized precedence constraints (problem $m, 1\left|\operatorname{gpr}, \delta_{n}, c_{j}\right| n p v$ or $P S|t e m p| \sum C_{j}^{F} \beta^{C_{j}}$ ). In our paper, we 
rely on a slightly modified version of this bi-directional generation scheme (BDGS) extended with a recursive forward/backward improvement method (FBIM) (Vanhoucke et al. (2001)) to increase the net present value. We test various priority rules implemented in the bi-directional generation scheme and develop a scatter search (SS) algorithm to solve the RCPSPDC. The outline of our paper is as follows. In the next section, we give an overview of the generation scheme used to solve the RCPSPDC. Furthermore, we discuss our specific implementation of the BDGS and its extension to the FBIM. In section 3, we outline the building blocks of our scatter search algorithm. In section 4 , we discuss detailed computational results for the BDGS with and without the FBIM and the SS algorithm. In order to motivate our choice for the scatter search methodology, we compare the performance of the algorithm with alternative metaheuristic formulations. We end with conclusions and ideas for future research avenues in section 5 .

\section{Schedule generation scheme}

\subsection{The bi-directional generation scheme}

The bi-directional priority-rule based method of Selle and Zimmermann (2003) consists of a simultaneously forward and backward approach and schedules at each iteration all eligible activities as soon (forward) or as late (backward) as possible with the precedence and renewable resource constraints. Hence, in each iteration an eligible activity is scheduled at its earliest or at its latest possible starting time given the partial schedule, based on a priority value represented by a random key vector element. The general idea is that an activity $i$ is forward eligible when all its predecessors have been scheduled (denoted by eligible activity $i(f)$ ) or backward eligible when all its successors have been scheduled (eligible activity $i(b))$. Since the generation scheme aims at scheduling eligible activities with positive cash flows as soon as possible and activities with negative cash flows as late as possible, the generation method considers the three following cases:

- If $c f_{i(f)} \geq 0$ : schedule $i(f)$ as soon as possible,

- If $c f_{i(b)} \leq 0$ : schedule $i(b)$ as late as possible,

- If $c f_{i(f)}<0$ and $c f_{i(b)}>0$ : schedule $i(f)$ as soon as possible when $h_{i(f)} \leq h_{i(b)}$ (defined hereunder) and schedule $i(b)$ as late as possible otherwise.

While the first two options are intuitively clear and directly contribute to the maximization of the net present value, the last option is counterintuitive. The last option either schedules an activity with negative cash flow as soon as possible or an activity with positive cash flow as late as possible, which is against the general philosophy of maximizing the net present value. Therefore, Selle and Zimmermann (2003) aim at minimizing the damage and calculate the $h_{i(f)}$ and $h_{i(b)}$ values as the financial loss arising when an activity is not scheduled at its earliest start time (positive cash flow activity $i(b)$ ) or at its latest start time (negative 
cash flow activity $i(f))$. To that purpose, they calculate the difference between the net present values when scheduling activity $i(f)$ at its latest possible starting time and scheduling it at its earliest starting time. Similarly, they calculate the difference between the net present value when scheduling activity $i(b)$ at its earliest and latest possible start time. The activity with the lowest difference will be selected and scheduled according to option 3 .

The bi-directional schedule generation scheme does not guarantee the construction of a schedule which ends within the pre-specified project deadline. Tight resource constraints and/or a low project deadline often result in an infeasible schedule (Selle and Zimmermann (2003)). In order to overcome this infeasibility problem, we test simple and straightforward extensions of the third option of the bi-directional generation scheme by implementing various other intuitive heuristic selection methods. More precisely, the third option is extended with two activity duration based, two resource based, two cash flows based and a random selection method, as follows:

- Activity Duration (AD): Assign the activity durations $d_{i(f)}$ and $d_{i(b)}$ to $h_{i(f)}$ and $h_{i(b)}$, respectively.

- Cumulative Activity Duration (CAD): Assign the activity duration $d_{i(f)}\left(d_{i(b)}\right)$ plus the durations of all its unscheduled successors (predecessors) to the value $h_{i(f)}$ (value $h_{i(b)}$ ). This heuristic in known in literature as the greatest ranked positional weight heuristic.

- Resource Demand (RD): Assign the work content $W_{i(f)}=\sum_{k=1}^{K} d_{i(f)} * r_{i(f) k}$ and $W_{i(b)}=\sum_{k=1}^{K} d_{i(b)} * r_{i(b) k}$ to $h_{i(f)}$ and $h_{i(b)}$, respectively.

- Cumulative Resource Demand (CRD): Assign the activity work content $W_{i(f)}\left(W_{i(b)}\right)$ plus the work content of all its unscheduled successors (predecessors) to the value $h_{i(f)}$ (value $\left.h_{i(b)}\right)$.

- $\quad$ Cash Flow (CF): Assign the cash flow values $-c f_{i(f)}$ and $c f_{i(b)}$ to $h_{i(f)}$ and $h_{i(b)}$, respectively. Note that this is a simplified version of Selle and Zimmermann (2003) since it ignores the time value of the activity cash flows and the time-span between their earliest and latest start time.

- Cumulative Cash Flow (CCF): Assign the cash flow values of activity $i(f)$ (activity $i(b)$ ) plus the cash flows of all its successors (predecessors) to the value $h_{i(f)}$ (value $h_{i(b)}$ ). This measure has been used by Baroum and Patterson (1996) under the name Cash Flow Weight in their single-pass cash flow weightbased procedure extended by a multi-pass shifting improvement algorithm.

- $\quad$ Random (RAN): Randomly generate a value for $h_{i(f)}$ and $h_{i(b)}$ from the interval $[0,1]$. This method boils down to the random selection of either $i(f)$ or $i(b)$ to be scheduled as soon as possible or as late as possible, respectively.

In the remainder of this paper, we distinguish between D-feasible (within the project deadline) and Dinfeasible (project duration larger than the deadline) schedules for which the D has been added to avoid confusion with resource infeasibilities. Although our scatter search algorithm calculates net present values 
for both D-feasible and D-infeasible solutions during its search process, it obviously only reports the net present value of a D-feasible solution as the best found solution at the end of the search. In section 3.1, the D-infeasibility problem is tackled by extending the subset generation method of the scatter search.

Moreover, D-feasible schedules can often be improved rather easily by shifting activities forwards or backwards. Baroum and Patterson (1996), for example, rely on a multi-pass forward/backward shifting algorithm which simply shifts activities with a positive (negative) cash flows to the project start (deadline) within their available slack. This shifting procedure is also implemented as an improvement technique in the generation scheme of Selle and Zimmermann (2003). Our improvement method to increase the net present value of D-feasible schedules relies on a recursive search method, which is discussed in the next sub-section.

\subsection{The recursive forward/backward improvement method}

Our improvement method is based on a recursive forward/backward method which is an extended version of the recursive method of Vanhoucke et al. (2001). The original method is developed to maximize the net present value of a resource-unconstrained project scheduling problem and aims at detecting sets of activities that can be shifted to increase the total project net present value. The method has been hybridized by principles and ideas from other research papers (such as Schwindt and Zimmermann (2001)) and has been proven to be very efficient by Vanhoucke (2006a). The extended recursive forward/backward improvement method differs from the original recursive search method in two ways:

1. The recursive search takes the renewable resource constraints into account: The original recursive search procedure of Vanhoucke et al. (2001) has been developed for maximizing the net present value of a project without the presence of renewable resource constraints (the so-called max-npv problem). The algorithm exploits the ideas of Grinold (1972) who stated that a solution for the max-npv problem can be represented by a sub-part of the project network representing a tree. The algorithm builds an earliest start schedule (with a corresponding tree) and aims at repetitively detecting sets of activities within the tree with a total negative net present value. Hence, shifting these activities towards the project deadline within the technological precedence relation results in an improved solution. This recursive search has been used to calculate lower bounds on the RCPSPDC at each node of a branch-and-bound algorithm of De Reyck and Herroelen (1998) and Vanhoucke et al. (2001). The forward/backward recursive search of the current manuscript relies on a similar logic but also takes the limited renewable resource availabilities into account. Both the construction of the initial tree and the shifts of sets of activities need to take these renewable resource constraints into account.

2. The recursive search alternates between a forward and backward step until no improvements can be found: The original recursive search procedure of Vanhoucke et al. (2001) relies on a forward approach 
which only allows shifts of sets of activities towards the project deadline. Hence, this approach requires an initial start tree where all activities (both with positive and negative cash flows) are scheduled as soon as possible. In our current manuscript, activity starting and finishing times are the result of the bidirectional schedule generation scheme, and are not necessarily earliest start schedules nor latest start schedules. Consequently, the modified recursive search of the current manuscript needs to be enhanced by a backward step in which the algorithm searches for sets of activities with a total positive net present value to shift towards the project start. The forward/backward recursive search algorithm of the current manuscript alternates between a forward step and a backward step until no further improvement (shifts) can be found.

Note that the recursive forward/backward improvement method can be applied using any generation scheme and is therefore not restricted to the use in combination with the bi-directional generation scheme. In the computational results section, we compare the bi-directional generation scheme with a forward and backward generation scheme, with and without the forward/backward improvement method. An illustrative example can be found in Vanhoucke (2006b).

\section{Scatter search procedure}

Scatter search is an evolutionary population-based method in which solutions are combined to yield better solutions using convex or non-convex linear combinations. Interesting references which describe the basic as well as more advanced features of the scatter search meta-heuristic have been presented in Glover (1998), Glover and Laguna (2000) and Marti et al. (2006). The pseudo-code for any general scatter search algorithm can be described as follows:

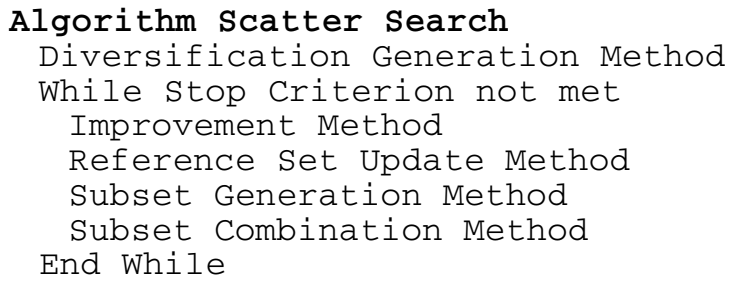

In the following sub-section, we describe our implementation of the scatter search approach to solve the RCPSPDC taking the various principles of section 2 into account. In section 3.2, we briefly discuss the dynamic update of three parameter values.

\subsection{Our scatter search implementation}


The Diversification Generation Method: In this initialization step, an initial pool of solutions is generated by randomly generating random key (RK) vectors and constructing the corresponding schedule using the bi-directional forward/backward generation scheme or the well-known serial schedule generation scheme. If the former generation scheme fails in constructing a schedule within the pre-defined deadline, the serial generation scheme is applied to generate a resource feasible schedule ignoring the pre-specified project deadline. As a result, this obtained schedule might end before, on or after the pre-specified project deadline. After the schedule generation, information from the obtained schedule will be used to transform the random key RK into a standardized random key (SRK) which fulfils the topological order condition of Valls et al. (2003). The implementation of the SRK value follows the four guidelines described in Debels and Vanhoucke (2007) and its use is based on the detected positive influence on the solution quality for the resource-constrained project scheduling problem.

The Improvement Method: This local search step aims at improving all elements from the pool of solutions, as follows:

- D-feasible solutions: these schedules are subject to the recursive forward/backward improvement method of section 2.2 in order to improve the total net present value of the schedules.

- D-infeasible solutions: these schedules with a project duration larger than the pre-defined project deadline are subject to the iterative forward/backward scheduling technique of $\mathrm{Li}$ and Willis (1992). In doing so, the algorithm tries to transform D-infeasible schedules into D-feasible schedules. If the resulting project duration is smaller than or equal to the pre-specified project duration, the obtained schedules are treated as D-feasible schedules, and hence, are the subject to the recursive forward/backward improvement procedure as mentioned above.

The Reference Update Method: A reference set is created containing high-quality (set $B_{1}$ ) and diverse (set $B_{2}$ ) solutions, with $B_{1} \cap B_{2}=\varnothing$, as follows:

- The quality subset $B_{1}$ : contains the best solutions found since the start of the procedure. This set only contains D-feasible schedules and has maximum $b_{1}$ solution elements. In order to guarantee that the best known solutions are diverse, a new schedule enters the subset $B_{1}$ only if the minimal distance to any existing element in the subset exceeds the threshold value $v_{1}$ or when the new candidate solution is better than any generated solution so far. The distance between two solutions $x$ and $y$ represented by their corresponding vectors $\mathrm{SRK}_{x}$ and $\mathrm{SRK}_{y}$ is measured as the sum of the absolute values of the component-wise difference between all vector elements of $\mathrm{SRK}_{x}$ and $\mathrm{SRK}_{y}$.

- The diversity subset $B_{2}$ : contains D-feasible solutions that are sufficiently different from the D-feasible solutions in subset $B_{1}$ and/or D-infeasible schedules. This set contains exactly $b_{2}$ solution elements (since the $B_{2}$ set also allows D-infeasible solutions, the number of elements is always equal to its 
maximal value). The divergence between D-feasible elements in $B_{1}$ and $B_{2}$ is guaranteed by a threshold value $v_{2}$ which is the minimal required distance between the candidate solution and any element of $B_{1}$. The parameter values $v_{1}$ and $v_{2}$ are dynamically updated throughout the search process, as explained in section 3.2. This two-tier design is maintained throughout the whole search of the procedure. While the $B_{1}$ subset contains the best known solutions so far, the $B_{2}$ subset is re-constructed from scratch during each run. Hence, all original elements are removed before the reference set update method begins.

The Subset Generation Method: The scatter search procedure operates on the reference set by combining pairs of solutions in a controlled way. The algorithm creates new solutions from all two-element subsets as follows:

- $B_{1} \times B_{1}$ : evaluation of all pairs of elements from $B_{1}$ containing at least one new solution compared to the previous generation. This method stimulates intensification since it selects two reference solutions from the same cluster.

- $B_{1} \times B_{2}$ : evaluation of all pairs combining an element from $B_{1}$ and an element from $B_{2}$. This method stimulates diversification since it selects two reference solutions from a different cluster.

- $B_{2} \times B_{2}$ : evaluation of all pairs of elements from $B_{2}$. This generation method is only executed when the number of solution elements in $B_{1}$ is lower than a threshold value $v_{3}$ (with $v_{3} \leq b_{1}$ ), and is particularly useful for project instances with a low deadline or tight resource constraints. In doing so, this method stimulates the generation of D-feasible solutions and aims at the increase of the number of solution elements in subset $B_{1}$. Unlike the dynamic update of the parameter values $v_{1}$ and $v_{2}, v_{3}$ is fixed throughout the search process (see section 3.2).

The Subset Combination Method: In order to combine solution elements from the different subsets, we have implemented two straightforward crossover operators, which are both used depending on the origin of the sets in the subset generation method.

- Two reference solutions from the same cluster $\left(B_{1} \times B_{1}\right.$ and $\left.B_{2} \times B_{2}\right)$ : The two-point crossover randomly selects two crossover points from the interval $c_{1} \in\left[0, n-c^{\min }\right]$ and $c_{2} \in\left[c_{1}+c^{\min }, n\right]$ with $c^{\min }$ the minimal number of activities subject to a change. Two child solutions are constructed by exchanging all SRK values between $c_{1}$ and $c_{2}$ between the parents. Activities that are not subject to a change are modified in order to preserve the relative ranking of these activities. More precisely, they get an SRK value equal to its original SRK value plus (minus) a large constant when its original value is higher (lower) than $c_{2}\left(c_{1}\right)$.

- Two reference solutions from a different cluster $\left(B_{1} \times B_{2}\right)$ : The cash flow crossover combines information from both parents into a single child solution as follows: the crossover operator scans all SRK values of the father and the mother and copies the lowest (largest) SRK value into the child solution when the cash flow of the corresponding activity is positive (negative). This approach aims at 
combining the best characteristics from two diverse solution elements, one from $B_{1}$ and another from the $B_{2}$ cluster with a minimal diversity of $v_{2}$.

\subsection{Dynamic parameter settings}

In section 3.1, we have defined three different threshold parameters each with a different function. The $v_{1}$ and $v_{2}$ parameters represent minimal distance values between two solutions while the $v_{3}$ is a parameter to guide the subset generation method. In this section, the values for the differend threshold parameters are discussed. Results are obtained by experimental tests on a large set of randomly generated project network instances generated by RanGen (Demeulemeester et al., 2003) with varying levels for the number of activities, the size of the acivity cash flows, the number of precedence relations in the network and the amount of resources each activity consumes. The chosen parameter values discussed in this section are selected based on the best average solution quality obtained.

The threshold parameter $v_{1}$ represents the minimal required distance between a candidate solution for subset $B_{1}$ and all existing solutions in $B_{1}$. In the beginning of the search process, the number of D-feasible elements in $B_{1}$ is low (initially equal to zero), and hence, the threshold needs to be set very low in order to allow the entrance of any D-feasible solution that is (sometimes only slightly) different from the current existing solutions. However, when the search process continues, the number of D-feasible elements in $B_{1}$ will likely to increase (up to its maximum of $b_{1}$ ), and hence, the algorithm need to increase the threshold value $v_{1}$ in order to guarantee more diverse high-quality schedules. However, the rate for which the number of solution elements in $B_{1}$ increases depends on factors such as the project deadline (finding D-feasible schedules within tight project deadlines is extremely complex) and the resource tightness. Once the number of D-feasible elements in $B_{1}$ is equal to its maximum value $b_{1}$, the threshold value $v_{1}$ can be decreased again depending on the number of new solution elements $b_{1}^{\text {new }}$ of $B_{1}$ during the previous run of the reference update method. In doing so, the algorithm continually increases or decreases its threshold value along its search. The threshold value can be calculated as $v_{1}=\frac{d i s t_{v_{1}}^{\max }-d i s t_{v_{1}}^{\min }}{b_{1}} * b_{1}^{\text {new }}$. The values for dist $t_{v_{1}}^{\min }$ ( dist $_{v_{1}}^{\max }$ ) represent the minimal (maximal) threshold values between which $v_{1}$ varies linearly and have been set to 1 and $2 * n$, respectively.

The threshold parameter $v_{2}$ represents the minimal required distance between a candidate solution of $B_{2}$ and any element of $B_{1}$. The dynamic calculation of the threshold value $v_{2}$ follows a similar reasoning as $v_{1}$ and depends on the number of D-feasible new solution elements $b_{2}^{\text {new }}$ in $B_{2}$ during the previous run of the reference update method. The threshold value can be calculated as $v_{2}=\frac{d i s t_{v_{2}}^{\max }-d i s t t_{v_{2}}^{\min }}{b_{2}} * b_{2}^{\text {new }}$. The values 
for dist $_{v_{2}}^{\min }$ ( dist $_{v_{2}}^{\max }$ ) represent the minimal (maximal) threshold values between which $v_{2}$ varies linearly and have been set to 1 and $10 * n$, respectively.

The threshold parameter $v_{3}$ represents the minimal number of solution elements in $B_{1}$ necessary to finish the $B_{2} \times B_{2}$ search in the subset generation method. In our implementation, we have set $v_{3}$ to a fixed value equal to $b_{1} / 3$.

\section{Computational results}

In this section, we test the performance of the different solution procedures on two randomly generated test sets consisting of resource-constrained problem instances generated by RanGen (Demeulemeester et al. 2003). Each project instance has been extended by activity cash flows and a project deadline. In section 4.1, the different versions of the bi-directional generation scheme and the recursive improvement method are tested by enumerating all possible random key values on small project instances of a first dataset. Test results show that neither the original bi-directional generation scheme, nor its straightforward extensions are able to produce optimal results, and the random factor is necessary to produce the best results. Section 4.2 reports results of the scatter search procedure, and compares the contribution of the generation scheme/improvement method on the solution quality. In section 4.3, the choice of the scatter search methodology is briefly motivated by showing computational results for alternative search procedures. All tests were carried out on a Dell computer with a Dual Core processor $2.8 \mathrm{Ghz}$ and $2 \mathrm{~Gb}$ RAM.

\subsection{Full enumeration}

In this section, we compare the performance of the various generation schemes on the first test set by enumerating all possible random keys that fulfil the topological order condition. Due to the huge amount of different possible keys, this experiment is restricted to project instances with 10 activities. The test instances have been generated by RanGen (Demeulemeester et al., 2003) as follows: each instance contains 10 non-dummy activities with each duration randomly generated between 1 and 10. Each project instance has an order strength OS (Mastor (1970)) and a resource-constrainedness RC (Patterson (1976)) fixed at $0.25,0.50$, or 0.75 . All project instances have 4 different resource types with availabilities of 10 units and have a resource use equal to two (each activity needs exact two of the four resources). The project deadline has been set to the minimal resource-constrained project deadline (obtained by the procedure of Demeulemeester and Herroelen (1992)) or to this minimal deadline exceeded by 5 time units. The cash flows have been generated between $[-500,500]$ such that the percentage of negative cash flows varies between $0 \%$ and $100 \%$ in steps of $10 \%$. Using 10 instances for each problem setting, we obtain a problem set of $3 * 3 * 2 * 11 * 10=1920$ problem instances. 
In order to measure the quality of the generation scheme under study, we calculate the average relative deviation between the resulting heuristic solutions $n p v^{\text {heur }}$ and the optimal solution $n p v^{\text {opt }}$ obtained by the procedure of Vanhoucke et al. (2001), as $\bar{\Delta}_{n p v}=\left|\frac{n p v^{o p t}-n p v^{h e u r}}{n p v^{o p t}}\right|$. Table 1 reports the results for the generation schemes (forward serial generation scheme (FOR), backward serial generation scheme (BAC) or bi-directional (all remaining columns)) with (yes) or without (no) the recursive improvement method of section 2.2. The bi-directional generation scheme has been implemented using the different third option rules of section 2.1. The table reveals that the simple forward and backward schedule generation scheme perform poor and generate heuristic solutions that deviate from the optimal solution with approximately $5 \%$ (without the recursive improvement method) and 3\% (with the recursive improvement method). Moreover, the results show that the use of the bi-directional scheme improves the results dramatically, although some versions are still not able to generate the optimal solution under full enumeration. The resource-based approach (RD and CRD) perform worse than the activity duration based approach (AD and CAD) which is, on its turn, outperformed by the cash flow based approach (CF and $\mathrm{CCF}$ ). The results for the original bidirectional generation scheme of Selle and Zimmermann (2003, column SZ) are very similar to the CF approach. Unfortunately, none of the straightforward extensions to the original SZ approach is able to always produce optimal results. This means that for some network instances, the third option will systematically select the wrong activity to be scheduled for any RK value, always leading to sub-optimal results. The results show that a simple modification to a random third option rule followed by a recursive improvement method leads to the best results. Finally, the table clearly shows that the use of the recursive improvement method leads to improved results for all approaches in the table.

\section{$<<$ insert table 1 about here $>>$}

Table 2 displays the average percentage of D-feasible solutions found per problem instance as the total number of feasible solutions found divided by the total number of evaluated priority vectors. Since we have found that the percentage negative cash flows has no significant influence on the number of D-feasible solutions, it is not included in table 2 .

\section{$<$ insert table 2 about here $>>$}

First, the table clearly reveals that infeasibilities occur more often when the order strength is low and the resource-constrainedness is high. Selle and Zimmermann (2003) have shown that their bi-directional generation scheme is not always able to generate D-feasible instances and the likelihood for infeasibilities increases with tight resource constraints. The decreasing complexity for the order strength has been noted by various authors (see e.g. Herroelen and De Reyck (1999) and Demeulemeester et al. (2003), amongst 
others). Second, the table shows that finding feasible solutions is harder for a project scheduling problem with a tight deadline. It is intuitively clear that a project instance with a strict deadline leads to more Dinfeasible schedules than a similar instance with more scheduling freedom. Finally, we note that the results differ only slightly between the different versions of all generation schemes. The minimal overall value is equal to $75.88 \%$ (the $\mathrm{AD}$ approach) while the maximal value equals $78.99 \%$ (the FOR approach). This is, however, not shown in table 2.

\subsection{Scatter search algorithm}

In this section, we present detailed computational results to test the performance of the scatter search algorithm and compare the obtained results with optimal or best known feasible solutions. Moreover, we present a randomly generated dataset containing 17,280 RCPSPDC instances that can be downloaded from our website for future research purposes.

The test instances have been generated by RanGen (Demeulemeester et al. 2003) under the settings displayed in table 3 . The project deadline has been set to the minimal resource-constrained project deadline exceeded by a certain percentage of this project duration (see table). In order to find the minimal project deadline, we have used the branch-and-bound procedure of Demeulemeester and Herroelen (1992) for the 25-activity instances and the decomposition-based genetic algorithm of Debels and Vanhoucke (2007) for all other instances truncated after 100,000 generated schedules. Hence, the minimal project deadlines for the 50,75 and 100-activity instances are not necessarily optimal. Using 10 instances for each problem setting, we obtain a problem set of $4 * 3 * 3 * 2 * 4 * 6 * 10=17,280$ problem instances.

\section{$<<$ insert table 3 about here $>>$}

Table 4 displays the results for the 25-activity instances and compares the solutions obtained by the branchand-bound procedure of Vanhoucke et al. (2001) with the heuristic solutions obtained by our scatter search procedures truncated after 5,000 generated schedules and by a random start heuristic. The branch-andbound procedure has been truncated after a pre-specified time limit of 100 seconds, which results in three classes of solutions: optimal, feasible and infeasible solutions. The optimal solutions have been found within the pre-specified time limit. The feasible solutions have been reported after truncation and cannot be proven to be optimal. If after the time limit no feasible solution can be found, this solution enters the class of infeasible solutions. The random start heuristic randomly generates 5,000 random key vectors that are transformed into a schedule by the bi-directional generation scheme and improved by the recursive forward/backward improvement method. The heuristic solutions obtained by the scatter search procedure and the multi-start heuristic are compared with all solutions from these three classes. Furthermore, we report whether the heuristic solution is worse (lower net present value, denoted by “-“), equal (“=”) or 
better (higher net present value, or "+") than the corresponding solution obtained by the BB procedure. The different runs correspond with different versions of the scatter search procedure, as follows:

- Run 1: Scatter search with iterative forward/backward algorithm of Li and Willis (1992)

- Run 2: Scatter search with iterative forward/backward algorithm of Li and Willis (1992) followed by the recursive forward/backward improvement method of section 2.2

- Run 3: Scatter search with the bi-directional generation scheme (with a random choice for the third option)

- Run 4: Scatter search with the bi-directional generation scheme (random choice) followed by the recursive forward/backward improvement method of section 2.2.

\section{$<$ insert table 4 about here $>>$}

The table reveals the following encouraging results. First, the comparison between the random start rows and the scatter search - run4 reveals that the scatter search procedure outperforms the random start heuristic (both procedures work with the bi-directional generation scheme (random choice) followed by the recursive forward/backward improvement method). Second, the scatter search procedure never leads to infeasible solutions, and the beneficial effect of the bi-directional generation scheme and the recursive improvement method is highlighted by the increasing number of solutions that are equal (better) than the solutions obtained by the truncated branch-and-bound procedure. While the run 1 version still has $36.57 \%+$ $32.94 \%=69.51 \%$ solutions that are worse that the $\mathrm{B} \& \mathrm{~B}$ solutions, the run 4 version has decreased that number to $13.89 \% .6 .74 \%(12.55 \%)$ of the solutions are equal to (better than) the feasible and optimal truncated B\&B solution for the run1 version, and this number increases to $37.09 \%$ (37.82\%) for the run 4 version. Last, note that the results are obtained after an average CPU time of 2.19 seconds, while the B\&B solutions have an average run time of 65.21 seconds (truncated after 100 seconds). Alternative computational experiments (which are not shown in the current paper) with larger project instances have revealed that the increases in the CPU time are relatively stable. As an example, project networks with 1,000 activities and an OS value of 0.5 and a RC value of 0.5 can be solved in - on the average - 19.78 seconds under a stop criterion of 5,000 generated schedules. Note that a schedule is counted each time the objective function (i.e. project's the net present value) is evaluated. Hence, one run of the recursive forward/backward procedure make consume as much schedules as the number of improvements found by shifting sets of activities.

\subsection{Comparison with alternative solution procedures}

Due to the endless supply of new scheduling methods for various scheduling problems, it is often difficult to claim fundamentally new ideas in the field of heuristic optimization or to establish their worth except by 
empirical testing. We realize that the problem under study can be solved by a variety of meta-heuristic procedures, and hence, no specific reason has been given yet for the specific choice of the scatter search algorithm. Based on results from previous research for other problem types (e.g. the enormous metaheuristic research efforts for the RCPSP, summarized in Kolisch and Hartmann (2006)) and temporary results obtained from a working paper for the RCPSPDC (Vanhoucke (2007)), we have developed various alternative meta-heuristic search procedures and compared them with each other. Hence, the presentation of the scatter search algorithm in the current manuscript is not a random choice, but rather the result of extensive comparative computational test runs between various alternative heuristic solution approaches. The main results of this extensive test run are briefly discussed in the current section, for which four alternative meta-heuristic search procedures have been taken into consideration, as follows:

SS(2): The scatter search procedure as described in section 3 containing a dual population of best and diverse solutions.

SS(3): The current scatter search procedure has been extended to three reference sets, each containing relevant and problem specific information. The set $B_{1}$ contains the best population elements, and is not different from SS(2). Reference sets $B_{2}^{+}$and $B_{2}^{-}$contain diverse solutions split up in two sub-populations. Each population contains the best diverse solution elements measured as the solution quality on a part of the schedule. More precisely, the reference set $B_{2}^{+}\left(B_{2}^{-}\right)$contains solutions elements ranked according to decreasing positive (negative) net present values measured on the set of positive (negative) cash flow activities. This modification is inspired by the triple population heuristic search procedure of Vanhoucke (2007) who has shown that the combination of elements from the sets $B_{2}^{+}$and $B_{2}^{-}$leads to further improvements while investigating the trade-off between a project's total duration and its corresponding net present value for the RCPSPDC.

GA: A single population genetic algorithm has been developed and tested with various crossover operators taken from literature (Kolisch and Hartmann (2006)), for which the best found combination of crossover operators are withhold.

EM: Inspired by the electromagnetic principles of Birbil and Fang (2003), an electromagnetic search procedure has been developed following the law of Coulomb to create new solutions from a pool of existing solutions. This technique has been proven its worth for the RCPSP in Debels and Vanhoucke (2006) and Debels et al. (2006).

The results have also been compared by an adapted version of the currently best performing algorithm from literature, the tabu search algorithm of Zhu and Padman (1999) (denoted by the column with label ZP). This tabu search procedure dynamically creates an initial solution based on six heuristics and gradually improves the solution(s) by swapping and insertion techniques, taking the principles of tabu search (restriction by means of a tabu list, aspiration criteria, multiple start solutions, ...) into account. We have 
tested this procedure under different settings and have run our results based on the best found settings. The authors show that their tabu search procedure outperforms many other procedures in literature. More precisely, they show that their tabu search approach dominates in $80 \%$ of the small size projects and in $94 \%$ of the large size projects.

Table 5 displays the solutions for the five different meta-heuristic search procedures truncated after 5,000 generated schedules. The column \%Feas displays the percentage of D-feasible solutions. The solution quality has been calculated as the average relative deviation (RDev) from the optimal net present value of the corresponding project scheduling problem instance ignoring the resource constraints (lower deviations denote a better solution quality). This so-called max-npv problem has been solved by the efficient recursive search method described in Vanhoucke (2006a). The average relative deviation is only calculated when all five solution algorithms have found a D-feasible solution, to ensure comparability between the RDev values. Results show that the scatter search procedure SS(2) clearly outperforms all other meta-heuristic search procedures, both in terms of solution quality and percentage of feasible solutions found. Note that four algorithms (i.e. GA, EM, SS(2) and SS(3)) incorporate both the bi-directional generation scheme and the recursive forward/backward improvement method while the tabu search procedure ZP follows a complete other schedule generation approach based on the tabu search principles.

\section{$<$ insert table 5 about here $>>$}

Note that the $b_{1}$ and $b_{2}$ values for the $\mathrm{SS}(2)$ algorithm depend on the stop criterion and have been set to 25 and 10 for 5,000 schedules (see table 5). Other parameters are stop criterion independent: the number of initial solution elements in the diversification generation method is always equal to 500 and the minimum number of activities subject to a change in the subset combination method equals $c^{\min }=n / 5$. These observations are in line with earlier scatter search results for the RCPSP described in Debels et al. (2006). We also tested the procedures with a 50,000 schedules stop criteration (with $b_{1}=50$ and $b_{2}=30$ ) leading to an improvement of the solution quality to $\mathrm{RDev}=186.45 \%, 221.34 \%, 219.36 \%, 237.89 \%$ and $268.72 \%$ for the SS(2), SS(3), GA, EM and ZP procedure. We recommend future researchers to test their procedures on the same benchmark set and to report their results in a similar way as in table 5. Note that we were not able to compare these results with other state-of-the-art procedures available in the open literature for two main reasons. First, none of the existing research papers uses a standard benchmark dataset and hence, we were not able to compare our results with best known solutions and secondly, many research papers use a slightly different activity and/or event cash flow assumption or payment structure, which makes the comparison of solutions irrelevant and/or impossible. However, we hope that comparison will be made easier in the future with the help of table 5 and the benchmark set proposed in the paper. Therefore, all detailed results, executables, test instances and detailed information can be downloaded from our website www.projectmanagement.ugent.be/npv.php. 


\section{Conclusions}

In this paper, we presented a scatter search algorithm to solve the resource-constrained project scheduling problem with discounted cash flows. This meta-heuristic procedure makes use of a bi-directional generation scheme and a recursive forward/backward improvement method and follows the principles of scatter search optimization. We have tested various variants of our algorithm on a self generated dataset containing 17,280 problem instances, have illustrated the contribution of the bi-directional generation scheme and the beneficial effect of the recursive forward/backward improvement method. In order to motivate the choice of both the problem description and the meta-heuristic methodology, we have compared the newly developed method with alternative heuristic solution procedures. In order to facilitate comparison for future research developments, we have reported best known solutions under three different stop criteria and created a website where all detailed information can be downloaded.

Our future intentions are as follows: First, we want to develop more advanced meta-heuristic search procedures to extend the basic problem type to, for example, multi-mode scheduling problems, pre-emptive activity execution, variable cash flows and many more. We believe that the bi-directional generation scheme and the recursive forward/backward improvement method can still be used for more advanced problem formulations. Second, we want to compare the scatter search framework with the building blocks of other meta-heuristics, such as genetic algorithms, particle swarm optimization, ant colony optimization, etc... and compare their performance on our proposed dataset.

\section{Acknowledgements}

We acknowledge the financial support from the FWO (Fonds voor Wetenschappelijk Onderzoek) Project under contract number G/0194/06.

\section{References}

Baroum, S.M. and Patterson, J.H., 1996, “The development of cash flow weight procedures for maximizing the net present value of a project", Journal of Operations Management, 14, 209-227.

Birbil, I., and Fang, S.C., 2003, “An electro-magnetism-like mechanism for global optimization”, Journal of Global Optimization 25, 263-282.

Blazewicz, J., Lenstra, J.K. and Rinnooy Kan, A.H.G., 1983, "Scheduling subject to resource constraints: classification and complexity", Discrete Applied Mathematics, 5, 11-24. 
Brücker, P., Drexl, A., Möhring, R., Neumann, K. and Pesch, E., 1999, "Resource-constrained project scheduling: notation, classification, models and methods", European Journal of Operational Research, $112,3-41$.

Debels, D. and Vanhoucke, M., 2006, “An Electromagnetism Meta-Heuristic for the Resource-Constrained Project Scheduling Problem”, Lecture Notes in Computer Science, 3871, 259-270.

Debels, D. and Vanhoucke, M., 2007, "A decomposition-based genetic algorithm for the resourceconstrained project scheduling problem", to appear in Operations Research.

Debels, D., De Reyck, B., Leus, R. and Vanhoucke, M., 2006, "A hybrid scatter search/electromagnetism meta-heuristic for project scheduling", European Journal of Operational Research, 169, 638-653.

Demeulemeester, E. and Herroelen, W., 1992, "A branch-and-bound procedure for the multiple resourceconstrained project scheduling problem", Management Science, 38, 1803-1818.

Demeulemeester, E., Vanhoucke, M., and Herroelen, W., 2003, “A random network generator for activityon-the-node networks", Journal of Scheduling, 6, 13-34.

De Reyck, B. and Herroelen, W., 1998, “Optimal procedure for the resource-constrained project scheduling problem with discounted cash flows and generalized precedence relations", Computers and Operations Research, 25, 1-17.

Glover, F, 1998, "A template for scatter search and path relinking", Lecture Notes in Computer Science, $1363,13-54$

Glover, F. and Laguna, M., 2000, "Fundamentals of scatter search and path relinking", Control and Cybernetics, 3, 653-684.

Grinold, R.C., 1972, “The payment scheduling problem”, Naval Research Logistics Quarterly, 19, 123-136.

Hartmann, S. and Kolisch, R., 2000, "Experimental evaluation of state-of-the-art heuristics for the resource-constrained project scheduling problem”, European Journal of Operational Research, 127, 394407.

Herroelen, W. and De Reyck, B., 1999, "Phase transitions in project scheduling", Journal of the Operational Research Quarterly, 50, 148-156.

Herroelen, W., Demeulemeester, E. and De Reyck, B., 1999, "A classification scheme for project scheduling. In: Weglarz, J. (Ed.), Project Scheduling - Recent Models, Algorithms and Applications", International Series in Operations Research and Management Science, Kluwer Academic Publishers, Boston, 14, 77-106.

Herroelen, W., De Reyck, B. and Demeulemeester, E., 1998, "Resource-constrained project scheduling: a survey of recent developments", Computers and Operations Research, 25, 279-302.

Icmeli, O., Erenguc, S.S. and Zappe, C.J., 1993, "Project scheduling problems: a survey", International Journal of Operations and Productions Management, 13, 80-91.

Kolisch, R. and Hartmann, S., 2006, "Experimental investigation of Heuristics for resource-constrained project scheduling: an update", European Journal of Operational Research, 174, 23-37. 
Kolisch, R. and Padman, R., 2001, “An integrated survey of deterministic project scheduling”, Omega, 49, 249-272.

Li, K.Y. and Willis, R.J., 1992, "An iterative scheduling technique for resource-constrained project scheduling”, European Journal of Operational Research, 56, 370-379.

Marti, R., Laguna, M. and Glover, F., 2006, “Principles of scatter search”, European Journal of Operational Research, 169, 359-372.

Mastor, A.A., 1970, "An experimental and comparative evaluation of production line balancing techniques", Management Science, 16, 728-746.

Mika, M., Waligora, G. and Weglarz, J., 2005, "Simulated annealing and tabu search for multi-mode resource-constrained project scheduling with positive discounted cash flows and different payment models", European Journal of Operational Research, 164, 639-668.

Özdamar, L. and Ulusoy, G., 1995, “A survey on the resource-constrained project scheduling problem”, IIE Transactions, 27, 574-586.

Patterson, J.H., 1976, "Project scheduling: the effects of problem structure on heuristic scheduling", Naval Research Logistics, 23, 95-123.

Schwindt, C. and Zimmermann, J., 2001, "A steepest ascent approach to maximizing the net present value of projects”, Mathematical Methods of Operations Research, 53, 435-450.

Selle, T. and Zimmermann, J., 2003, "A bidirectional heuristic for maximizing the net present value of large-scale projects subject to limited resources”, Naval Research Logistics, 50, 130-148.

Valls, V., Quintanilla, S., Ballestín, F., 2003. Resource-constrained project scheduling: a critical activity reordering heuristic, European Journal of Operational Research, 149, 282-301.

Vanhoucke, M., 2006a, “An efficient hybrid search procedure for various optimization problems", Lecture Notes on Computer Science, 3906, 272-283.

Vanhoucke, M., 2006b, "A scatter search procedure for maximizing the net present value of a resourceconstrained project with fixed activity cash flows", working paper 06/417, Ghent University.

Vanhoucke, M., 2007, "A genetic algorithm to investigate the trade-off between project lead time and net present value", working paper 07/456, Ghent University (under submission).

Vanhoucke, M., Demeulemeester, E. and Herroelen, W., 2001, "On maximizing the net present value of a project under renewable resource constraints", Management Science, 47, 1113-1121.

Zhu, D. and Padman, R., 1999, "A metaheuristic scheduling procedure for resource-constrained projects with cash flows", Naval Research Logistics, 46, 912-927. 
Table 1. Relative deviation $\bar{\Delta}_{n p v}$ from the optimal solution (in \%)

\begin{tabular}{|c|c|c|c|c|c|c|c|c|c|c|c|c|c|c|c|c|c|c|c|c|}
\hline & & \multicolumn{2}{|c|}{ AD } & \multicolumn{2}{|c|}{ CAD } & \multicolumn{2}{|c|}{ RD } & \multicolumn{2}{|c|}{ CRD } & \multicolumn{2}{|c|}{ CF } & \multicolumn{2}{|c|}{ CCF } & \multicolumn{2}{|c|}{ RAN } & \multicolumn{2}{|c|}{ FOR } & \multicolumn{2}{|c|}{ BAC } & \multirow[t]{2}{*}{ SZ } \\
\hline & & no & yes & no & yes & no & yes & no & yes & no & yes & no & yes & no & yes & no & yes & no & yes & \\
\hline Overall & & 0.133 & 0.005 & 0.310 & 0.036 & 0.766 & 0.065 & 0.321 & 0.020 & 0.036 & 0.020 & 0.123 & 0.023 & .005 & .000 & 4.668 & 2.247 & 5.763 & 3.159 & 0.035 \\
\hline \multirow{3}{*}{ OS } & 0.25 & 0.108 & 0.008 & 0.251 & 0.061 & 0.709 & 0.105 & 0.369 & 0.020 & 0.013 & 0.000 & 0.118 & 0.046 & .000 & 0.000 & 5.359 & 2.361 & 6.826 & 3.552 & 0.012 \\
\hline & 0.50 & 0.081 & 0.005 & 0.345 & 0.029 & 0.695 & 0.074 & 0.289 & 0.010 & 0.062 & 0.037 & 128 & 0.018 & .013 & 0.000 & .920 & 1.936 & 5.243 & 2.941 & 0.063 \\
\hline & 0.75 & 0.209 & 0.003 & 0.334 & 0.019 & 0.895 & 0.015 & 0.303 & 0.028 & 0.034 & 0.024 & 0.123 & 0.006 & 0.002 & 0.000 & 3.725 & 2.445 & 5.221 & 2.985 & 0.034 \\
\hline \multirow{3}{*}{$\mathrm{RC}$} & 0.25 & 0.194 & 0.000 & 0.382 & 0.001 & 1.035 & 0.004 & 0.551 & 0.001 & 0.017 & 0.000 & 0.149 & 0.000 & 0.004 & 0.000 & 6.975 & 1.954 & 8.754 & 3.219 & 0.016 \\
\hline & 0.50 & 0.124 & 0.011 & 0.324 & 0.085 & 0.734 & 0.155 & 0.246 & 0.045 & 0.015 & 0.001 & 0.130 & 0.052 & 0.000 & 0.000 & 4.390 & 2.863 & 5.666 & 3.748 & 0.014 \\
\hline & 0.75 & 0.080 & 0.004 & 0.224 & 0.023 & 0.530 & 0.034 & 0.166 & 0.013 & 0.078 & 0.061 & 0.090 & 0.018 & 0.010 & 0.000 & 2.639 & 1.926 & 2.870 & 2.511 & 0.077 \\
\hline \multirow{2}{*}{ Deadline } & 0 & 0.010 & 0.003 & 0.039 & 0.014 & 0.153 & 0.037 & 0.083 & 0.001 & 0.029 & 0.025 & 0.033 & 0.022 & 0.001 & 0.000 & 2.988 & 0.566 & 3.320 & 0.922 & 0.028 \\
\hline & 5 & 0.256 & 0.008 & 0.581 & 0.058 & 1.379 & 0.093 & 0.559 & 0.039 & 0.044 & 0.016 & 0.213 & 0.024 & 0.009 & 0.000 & 6.348 & 3.929 & 8.207 & 5.396 & 0.043 \\
\hline \multirow{11}{*}{$\%$ Neg } & 0 & 0.000 & 0.000 & 0.000 & 0.000 & 0.000 & 0.000 & 0.000 & 0.000 & 0.000 & 0.000 & 0.000 & 0.000 & 0.000 & 0.000 & 0.000 & 0.000 & 3.957 & 1.956 & 0.000 \\
\hline & 10 & 0.000 & 0.000 & 0.000 & 0.000 & 0.000 & 0.000 & 0.000 & 0.000 & 0.000 & 0.000 & 0.000 & 0.000 & 0.000 & 0.000 & 1.592 & 0.847 & 4.554 & 2.591 & 0.000 \\
\hline & 20 & 0.000 & 0.000 & 0.000 & 0.000 & 0.000 & 0.000 & 0.000 & 0.000 & 0.000 & 0.000 & 0.000 & 0.000 & 0.000 & 0.000 & 1.592 & 0.847 & 4.554 & 2.591 & 0.000 \\
\hline & 30 & 0.000 & 0.000 & 0.000 & 0.000 & 0.000 & 0.000 & 0.000 & 0.000 & 0.000 & 0.000 & 0.000 & 0.000 & 0.000 & 0.000 & 1.592 & 0.847 & 4.554 & 2.591 & 0.000 \\
\hline & 40 & 0.125 & 0.004 & 0.171 & 0.011 & 0.658 & 0.048 & 0.293 & 0.021 & 0.035 & 0.026 & 0.091 & 0.018 & 0.004 & 0.000 & 4.075 & 1.962 & 6.214 & 3.554 & 0.034 \\
\hline & 50 & 0.125 & 0.004 & 0.171 & 0.011 & 0.658 & 0.048 & 0.293 & 0.021 & 0.035 & 0.026 & 0.091 & 0.018 & 0.004 & 0.000 & 4.075 & 1.962 & 6.214 & 3.554 & 0.034 \\
\hline & 60 & 0.125 & 0.004 & 0.171 & 0.011 & 0.658 & 0.048 & 0.293 & 0.021 & 0.035 & 0.026 & 0.091 & 0.018 & 0.004 & 0.000 & 4.075 & 1.962 & 6.214 & 3.554 & 0.035 \\
\hline & 70 & 0.446 & 0.018 & 1.244 & 0.166 & 2.945 & 0.262 & 1.158 & 0.055 & 0.128 & 0.070 & 0.449 & 0.090 & 0.020 & 0.000 & 10.705 & 4.900 & 11.943 & 6.221 & 0.128 \\
\hline & 80 & 0.446 & 0.018 & 1.244 & 0.166 & 2.945 & 0.262 & 1.158 & 0.055 & 0.128 & 0.070 & 0.449 & 0.090 & 0.020 & 0.000 & 10.705 & 4.900 & 11.943 & 6.221 & 0.127 \\
\hline & 00 & 0.193 & 0.007 & 0.407 & 0.032 & 0.565 & 0.044 & 0.333 & 0.042 & 0.038 & 0.007 & 0.183 & 0.022 & 0.004 & 0.000 & 8.432 & 4.262 & 3.248 & 1.920 & 0.037 \\
\hline & 100 & 0.000 & 0.000 & 0.000 & 0.000 & 0.000 & 0.000 & 0.000 & 0.000 & 0.000 & 0.000 & 0.000 & 0.000 & 0.000 & 0.000 & 4.503 & 2.232 & 0.000 & 0.000 & 0.000 \\
\hline
\end{tabular}


Table 2. Average percentage of D-feasible solutions per problem instance

\begin{tabular}{|cc|ccc|ccc|}
\hline & & \multicolumn{7}{|c|}{ RC } \\
& & 0.25 & 0.50 & 0.75 & 0.25 & 0.50 & 0.75 \\
\hline Deadline & & \multicolumn{3}{|c|}{0} & & \multicolumn{3}{c|}{5} \\
\hline \multirow{3}{*}{ OS } & 0.25 & 85.26 & 38.12 & 22.71 & 99.78 & 82.77 & 70.98 \\
& 0.50 & 95.38 & 49.37 & 37.12 & 100.00 & 90.45 & 73.44 \\
& 0.75 & 100.00 & 66.98 & 72.85 & 100.00 & 93.46 & 94.19 \\
\hline
\end{tabular}


Table 3. Parameter settings used to generate the test instances for the RCPSPDC

\begin{tabular}{ll}
\hline Numer of activities & $25,50,75$ or 100 \\
Activity durations & Randomly selected from the interval [1, 10] \\
Order strength OS & $0.25,0.50$ or 0.75 \\
Number of resource types & 4 \\
Resource constrainedness RC & $0.25,0.50$ or 0.75 \\
Resource use RU & 2 or 4 \\
Project deadline & $5,10,15$ or 20 \\
Discount rate $\alpha$ & 0.01 \\
Percentage negative cash flows & $0,20,40,60,80$ or 100 \\
\hline
\end{tabular}


Table 4. Computational results for the 25 activity networks

\begin{tabular}{|c|c|c|c|c|c|c|}
\hline & & & & \multicolumn{3}{|c|}{ B\&B } \\
\hline & & & & infeasible & feasible & optimal \\
\hline & & & & $11.20 \%$ & $49.86 \%$ & $38.94 \%$ \\
\hline \multirow{3}{*}{ 흥 } & \multirow{3}{*}{ 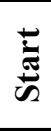 } & - & $60.30 \%$ & $x$ & $36.97 \%$ & $23.33 \%$ \\
\hline & & $=$ & $15.95 \%$ & $0.23 \%$ & $0.12 \%$ & $15.60 \%$ \\
\hline & & + & $23.75 \%$ & $10.97 \%$ & $12.78 \%$ & $x$ \\
\hline \multirow{12}{*}{ 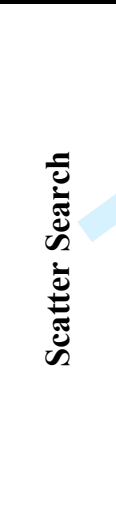 } & \multirow{3}{*}{$\bar{\Xi}$} & - & $69.51 \%$ & $x$ & $36.57 \%$ & $32.94 \%$ \\
\hline & & $=$ & $6.74 \%$ & $0.00 \%$ & $0.74 \%$ & $6.00 \%$ \\
\hline & & + & $23.75 \%$ & $11.20 \%$ & $12.55 \%$ & $x$ \\
\hline & \multirow{3}{*}{$\stackrel{N}{\Xi}$} & - & $67.36 \%$ & $x$ & $35.21 \%$ & $32.15 \%$ \\
\hline & & $=$ & $7.52 \%$ & $0.00 \%$ & $0.74 \%$ & $6.78 \%$ \\
\hline & & + & $25.12 \%$ & $11.20 \%$ & $13.91 \%$ & $x$ \\
\hline & \multirow{3}{*}{$\stackrel{m}{\Xi}$} & - & $21.23 \%$ & $x$ & $7.66 \%$ & $13.56 \%$ \\
\hline & & $=$ & $30.23 \%$ & $0.00 \%$ & $4.86 \%$ & $25.37 \%$ \\
\hline & & + & $\mathbf{4 8 . 5 4 \%}$ & $11.20 \%$ & $37.34 \%$ & $\times$ \\
\hline & \multirow{3}{*}{$\underset{\Xi}{ \pm}$} & - & $13.89 \%$ & $x$ & $5.95 \%$ & $7.94 \%$ \\
\hline & & $=$ & $37.08 \%$ & $0.00 \%$ & $6.09 \%$ & $31.00 \%$ \\
\hline & & + & $49.03 \%$ & $11.20 \%$ & $37.82 \%$ & $x$ \\
\hline
\end{tabular}

Table 5. Comparative computational tests and best known solutions

\begin{tabular}{|c|c|c|c|c|c|c|c|c|c|c|c|}
\hline & & \multicolumn{10}{|c|}{5,000 generated schedules } \\
\hline & & \multicolumn{2}{|c|}{$\operatorname{SS}(2)^{1}$} & \multicolumn{2}{|c|}{$\mathrm{SS}(3)^{2}$} & \multicolumn{2}{|c|}{$\mathbf{G A}^{3}$} & \multicolumn{2}{|c|}{$\mathbf{E M}^{4}$} & \multicolumn{2}{|c|}{$\mathbf{Z P}^{5}$} \\
\hline & & RDev & $\%$ Feas & RDev & $\%$ Feas & RDev & $\%$ Feas & RDev & $\%$ Feas & RDev & $\%$ Feas \\
\hline Overall & & 200.82 & $99.8 \%$ & 232.32 & $93.4 \%$ & 230.12 & $93.7 \%$ & 245.67 & $93.9 \%$ & 290.65 & $99.8 \%$ \\
\hline \multirow{4}{*}{ Act } & 25 & 191.34 & $100.0 \%$ & 205.57 & $99.8 \%$ & 206.82 & $99.8 \%$ & 222.30 & $99.8 \%$ & 253.35 & $100.0 \%$ \\
\hline & 50 & 282.37 & $99.9 \%$ & 343.56 & $96.1 \%$ & 340.92 & $96.3 \%$ & 343.38 & $96.5 \%$ & 399.59 & $99.9 \%$ \\
\hline & 75 & 146.56 & $99.8 \%$ & 170.18 & $90.5 \%$ & 165.78 & $91.1 \%$ & 192.56 & $91.4 \%$ & 218.94 & $99.7 \%$ \\
\hline & 100 & 183.02 & $99.7 \%$ & 209.95 & $87.0 \%$ & 206.96 & $87.6 \%$ & 224.43 & $87.9 \%$ & 290.70 & $99.7 \%$ \\
\hline \multirow{3}{*}{ OS } & 0.25 & 156.44 & $99.7 \%$ & 181.48 & $92.5 \%$ & 177.44 & $92.9 \%$ & 209.47 & $93.2 \%$ & 246.28 & $99.7 \%$ \\
\hline & 0.50 & 193.84 & $99.7 \%$ & 215.44 & $91.7 \%$ & 214.50 & $91.9 \%$ & 235.01 & $92.3 \%$ & 276.45 & $99.8 \%$ \\
\hline & 0.75 & 252.18 & $100.0 \%$ & 300.03 & $95.9 \%$ & 298.41 & $96.3 \%$ & 292.54 & $96.3 \%$ & 349.20 & $100.0 \%$ \\
\hline \multirow{3}{*}{$\mathrm{RC}$} & 0.25 & 61.42 & $99.7 \%$ & 67.97 & $91.1 \%$ & 65.33 & $91.7 \%$ & 84.65 & $92.0 \%$ & 120.44 & $99.9 \%$ \\
\hline & 0.50 & 322.27 & $99.7 \%$ & 382.96 & $91.8 \%$ & 379.39 & $92.2 \%$ & 388.53 & $92.5 \%$ & 465.06 & $99.6 \%$ \\
\hline & 0.75 & 218.77 & $100.0 \%$ & 246.01 & $97.1 \%$ & 245.64 & $97.3 \%$ & 263.83 & $97.2 \%$ & 286.44 & $100.0 \%$ \\
\hline \multirow{2}{*}{ RU } & 2 & 235.25 & $99.8 \%$ & 278.84 & $92.1 \%$ & 276.10 & $92.6 \%$ & 286.69 & $92.9 \%$ & 348.72 & $99.7 \%$ \\
\hline & 4 & 166.39 & $99.8 \%$ & 185.79 & $94.6 \%$ & 184.14 & $94.8 \%$ & 204.65 & $95.0 \%$ & 232.57 & $99.9 \%$ \\
\hline \multirow{4}{*}{ Deadline } & 5 & 174.71 & $99.3 \%$ & 193.76 & $75.4 \%$ & 194.73 & $76.4 \%$ & 208.54 & $77.0 \%$ & 283.85 & $99.3 \%$ \\
\hline & 10 & 146.53 & $100.0 \%$ & 163.44 & $98.1 \%$ & 160.96 & $98.5 \%$ & 179.96 & $98.6 \%$ & 197.10 & $100.0 \%$ \\
\hline & 15 & 332.34 & $100.0 \%$ & 398.27 & $100.0 \%$ & 395.44 & $100.0 \%$ & 401.37 & $100.0 \%$ & 465.26 & $100.0 \%$ \\
\hline & 20 & 149.71 & $100.0 \%$ & 173.79 & $100.0 \%$ & 169.36 & $100.0 \%$ & 192.81 & $100.0 \%$ & 216.38 & $100.0 \%$ \\
\hline \multirow{6}{*}{$\%$ Neg } & 0 & 29.10 & $99.8 \%$ & 30.81 & $94.7 \%$ & 30.72 & $95.3 \%$ & 30.83 & $94.5 \%$ & 31.43 & $99.8 \%$ \\
\hline & 20 & 26.55 & $99.8 \%$ & 27.92 & $93.4 \%$ & 27.86 & $93.6 \%$ & 30.31 & $94.1 \%$ & 30.55 & $99.8 \%$ \\
\hline & 40 & 64.94 & $99.8 \%$ & 72.18 & $92.9 \%$ & 71.10 & $93.2 \%$ & 82.88 & $93.7 \%$ & 93.74 & $99.8 \%$ \\
\hline & 60 & 454.10 & $99.8 \%$ & 545.95 & $92.7 \%$ & 541.41 & $93.3 \%$ & 553.96 & $93.9 \%$ & 670.76 & $99.8 \%$ \\
\hline & 80 & 389.01 & $99.8 \%$ & 434.66 & $93.3 \%$ & 428.01 & $93.1 \%$ & 497.72 & $93.6 \%$ & 593.11 & $99.8 \%$ \\
\hline & 100 & 241.23 & $100.0 \%$ & 282.36 & $93.2 \%$ & 281.63 & $93.9 \%$ & 278.32 & $93.6 \%$ & 324.30 & $99.8 \%$ \\
\hline
\end{tabular}

\footnotetext{
Results obtained from the scatter search algorithm described in the current manuscript

${ }^{2}$ Results obtained from a modified scatter search algorithm with three reference sets

${ }^{3}$ Results obtained from a genetic algorithm tested under different crossover operators

${ }^{4}$ Results obtained from an electromagnetic algorithm based on the principles of Birbil and Fang (2003)

${ }^{5}$ Results obtained from an adapted tabu search procedure of Zhu and Padman (1999)
} 
